\title{
A simple predictor of interface orientation of mesogenic fluids and its implications for organic semiconductors ${ }^{\dagger}$
}

\begin{abstract}
Belinda J. Boehm and David M. Huang*
From classical molecular dynamics simulations, we identify a simple and general predictor of molecular orientation at solid and vapour interfaces of isotropic fluids of anisotropic particles based on their shape and interaction anisotropy. For a wide variety of inter-particle interactions, temperatures, and substrate types within the range of typical organic semiconductors and their processing conditions, we find remarkable universal scaling of the orientation at the interface with the free energy calculated from pair interactions between close-packed nearest neighbours and an empirically derived universal relationship between the entropy and the shape anisotropy and bulk volume fraction of the fluid particles. The face-on orientation of fluid particles at the solid interface is generally predicted to be the equilibrium structure, although the alignment can be controlled by tuning the particle shape and substrate type, while changing the strength of fluid-fluid interactions is likely to play a less effective role. At the vapour interface, only the side-on structure is predicted, and conditions for which the face-on structure may be preferred, such as low temperature, low interaction anisotropy, or low shape anisotropy, are likely to result in little orientation preference (due to the low anisotropy) or be associated with a phase transition to an anisotropic bulk phase for systems with interactions in the range of typical organic semiconductors. Based on these results, we propose a set of guidelines for the rational design and processing of organic semiconductors to achieve a target orientation at a solid or vapour interface.
\end{abstract}

\section{Introduction}

Anisotropic molecules, whose shape deviates significantly from spherical, are hugely important in many technologies. Liquid crystal molecules, for example, form ordered phases that can be controlled by temperature or concentration, and are the basis of a multi-billion dollar display industry, with an extensive history dating back to the late nineteenth century. ${ }^{1}$ Additionally, organic molecules with extended $\pi$-conjugation, many of which can also be classified as liquid crystals, have been shown to display interesting opto-electronic properties, and devices based on these organic semiconductors (OSCs) are growing in importance as their performance improves. ${ }^{2,3}$

As important electronic processes often happen in the vicinity of interfaces, ${ }^{4-7}$ controlling the alignment of molecules at these interfaces is important for optimising the performances of OSC-based devices and liquid-crystal displays (LCDs). Due to the anisotropic shape of these molecules, symmetry breaking at both solid and vapour interfaces often leads to a preferred orientation, ${ }^{8}$ even when the bulk phase is isotropic. This interface alignment has broad implications for device performance.

For a uniaxial molecule (in which two of the principal axes are equivalent), alignment can vary between the extremes of the non-equivalent molecular axis aligned parallel (planar anchoring; side-on orientation for an oblate ellipsoid) or perpendicular (homeotropic anchoring; face-on orientation for an oblate ellipsoid) to the interface (Fig. 1). For biaxial molecules (in which all

Department of Chemistry, School of Physical Sciences, The University of Adelaide, Adelaide, Australia, Tel: +61 88313 5580; Email: david.huang@adelaide.edu.au $\dagger$ Electronic Supplementary Information (ESI) available. See DOI: 00.0000/00000000.
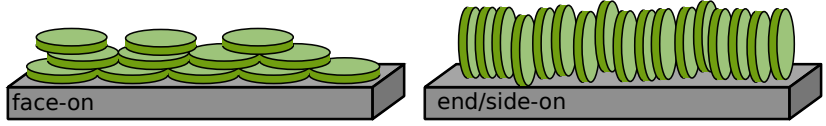

Fig. 1 Face-on versus end/side-on alignment at an interface for diskshaped particles. Interface orientation of rod-like particles can be similarly defined by the angle of their long axis with respect to the interface. For uniaxial particles, end-on and side-on orientations are equivalent.

three principal axes are different), three alignment extremes are possible: face-on, end-on, and side-on.

In OSCs in particular, orientation at the solid and vapour interface is important for improving the performance of a variety of devices, with different functionalities requiring different alignments. In organic field-effect transistors (OFETs), for example, charge mobility is greater when molecules are aligned side-on at the solid dielectric interface, as charge transport can occur in the $\pi$-stacking direction rather than having to proceed through the often insulating side-chains. ${ }^{9-14}$ Conversely, in organic photovoltaics (OPVs), the face-on orientation at the electron donoracceptor bulk heterojunction (BHJ) interface is favoured as it reduces charge recombination by allowing efficient charge transport away from the interface, while also ensuring better charge generation by increasing donor-acceptor orbital overlap. ${ }^{15-19}$ Organic light-emitting diode (OLED) performance also depends on the orientation of the emitter's transition dipole moment with respect to the substrate, with planar alignment preferred. ${ }^{20-23}$ The design of molecules for these devices should therefore focus not only on the electronic properties of the individual molecule, but also on how the molecules align at important interfaces in the device in order to maximise performance. 
Although the importance of controlling interface orientation in OSC devices is generally well understood, and there is no shortage of experimental ${ }^{16-18,24}$ or computational $^{25-30}$ examples of preferential alignment of anisotropic molecules at interfaces, there remain few general rules for predicting alignment at either the solid or vapour interface from the chemical structure of OSC molecules. We have recently extensively reviewed the subject in the context of OSCs, ${ }^{31}$ and despite a number of factors being implicated in controlling molecular orientation, in many cases an understanding of the general physical principles that dictate this behaviour was lacking, especially at the solid interface.

There is an extensive history of simulations of liquid crystals using simple computational models (see ref 32 for a review), which have been used to provide a basic understanding of the behaviour of these anisotropic molecules. A smaller subset of these studies have examined the interplay of repulsive and attractive interactions in controlling the interfacial orientation. At a free interface (such as that with a vapour phase), the orientation of anisotropic particles has been studied at various levels, ranging from mean field theories, ${ }^{33}$ to particle-based molecular dynamics (MD), ${ }^{34}$ and Monte-Carlo (MC) ${ }^{35,36}$ simulations. It has been found that switching between the face-on and side-on orientation at this interface can be achieved through tuning the anisotropy of the attractive interactions. In the absence of attractive interactions, the long axis of an anisotropic molecule has been predicted to align perpendicular to the interface due to excluded-volume entropic effects. ${ }^{33}$ MD simulations of the Gay-Berne (GB) fluid have indicated a dependence of the orientation at the vapour interface of nematic fluids on the ratio of the shape anisotropy to the interaction anisotropy of the particles. ${ }^{34,36}$ Fluids that are isotropic in the bulk have also been shown to display preferential alignment at a vapour interface. ${ }^{35}$

In contrast, at the solid interface, free volume is maximised in the face-on orientation, for which the long molecular axis is parallel to the interface. ${ }^{37}$ Density functional theory calculations of hard platelets, ${ }^{38,39}$ and theoretical calculations of hard spherocylinders 40 and spheroplatelets ${ }^{41}$ at hard impenetrable walls show a preference for alignment with the short axis of the molecule perpendicular to the wall due to entropic effects and minimisation of the surface tension. The inclusion of attractive interactions between fluid particles has been predicted to introduce a temperature dependence to interface orientation, with systems at high temperatures showing the same alignment as the purely repulsive systems due to the dominance of entropy, and a transition to the opposite alignment at lower temperatures as energetic effects become more significant. ${ }^{42,43}$ Many computational studies of specific systems have shown similar dependences on temperature and interaction anisotropy. ${ }^{44-49}$ In general, the orientation at the solid interface depends on both the strength and anisotropy of the fluid-substrate interactions, ${ }^{43}$ and the strength of the fluid-fluid interactions. ${ }^{50}$ A number of experimental studies report control of orientation at this interface for specific small molecules, ${ }^{19,44,46,51-55}$ and the strength of the interactions with the substrate has been shown to directly influence the orientation, again for a specific set of interaction parameters. 45

An interesting illustration of the contrasting behaviour of hard particles at solid and vapour interfaces is the case of a penetrable wall, from which a particle's centre-of-mass is repelled but which the rest of the molecule can penetrate to varying degrees. ${ }^{56,57}$ As the wall interacts isotropically with the centre-of-mass of the anisotropic particle, a highly penetrable wall is qualitatively similar to a free interface, whereas a impenetrable wall represents a solid interface. MC and density functional theory calculations of these interfaces show a transition between face-on alignment at an impenetrable surface and side-on alignment at a fully penetrable wall, ${ }^{56,57}$ consistent with previous theoretical predictions at solid and vapour interfaces, respectively, due to excluded-volume entropic effects. ${ }^{33,42}$

Although a number of general rules that appear to be relatively predictive for determining the orientation of anisotropic particles at both solid and vapour interfaces exist, and many specific examples of orientation control can be found in the literature, a more detailed understanding of how molecular shape and interactions control the orientation in general, and the ability to predict orientation based on combined energetic and entropic contributions using a simple analytical expression, is lacking. In this work, we investigate a series of OSC-like systems using equilibrium coarse-grained MD simulations of GB particles between solid and vapour-like interfaces. These simulations allow for systematic tuning of the strength of the interactions, shape of the anisotropic molecule, and its interactions with the substrate, to gain an understanding of how the interplay of these features influences the orientation at both the solid and vapour interfaces. The use of GB particles, which have a simple ellipsoidal shape, means that these results should be generally applicable to a wide range of molecules, whether OSCs or not, that share similar shapes and interactions, rather than specific to a single type or class of chemical structure.

We focus our work here on systems that are isotropic in the bulk in order to isolate the effects of the interfaces, to remove the additional complications associated with alignment of additional layers, and to broadly understand what happens under experimental conditions in which a thin film of anisotropic molecules is annealed from the melt, deposited from solution, or subjected to high operating temperatures. Generalisation to interfaces with bulk fluids that are anisotropic is possible. We also focus on simulations of equilibrium fluid structure. While thin-films in OSC devices are commonly deposited under non-equilibrium conditions, systems will evolve towards equilibrium over time. As device performance depends strongly on interface orientation, a shift away from a desired non-equilibrium morphology towards the thermodynamic minimum may result in a reduction in performance over time. It is therefore desirable for devices to have an as-deposited morphology that is close to equilibrium, as this will confer higher thermal stability on device performance. It is important then to understand the equilibrium behaviour of such molecules in order to develop materials that continue to perform well over the entire device lifetime.

The paper is organised as follows. We first describe the simulation and analysis methods in section 2 . We then show that molecular orientation of anisotropic fluid particles with both repulsive and attractive interactions at the solid and vapour interface of an 
isotropic fluid cannot generally be predicted using a previously proposed metric, and propose a semi-empirical mean-field estimate of the free-energy difference between face-on and side-on orientations as an alternative. We use simulations of purely repulsive particles confined between a solid and vapour interface to obtain quasi-universal relationships for the scaling of the entropic component of the free energy with the shape anisotropy and bulk density of the fluid particles (section 3.1). Combining this estimate of the entropy with an estimate of the energetic component of the free energy from nearest-neighbour pair interactions (section 3.2), we show that this free energy parameter accurately predicts molecular orientation at the solid (section 3.3.1) and vapour (section 3.3.2) interfaces. In section 3.4 we generalise these results into a number of practical design principles for OSCs, with the goal of providing guidelines towards rational design of OSC interfaces.

\section{Methods}

The orientation of a fluid of anisotropic particles at both solid (impenetrable) and vapour-like (penetrable) interfaces was studied using classical molecular dynamics (MD) simulations. A range of uniaxial oblate Gay-Berne (GB) ellipsoids, representative of typical small OSC molecules, were studied to elucidate the general effects of shape and interaction anisotropy. These simple models greatly increase computational efficiency compared with all-atom simulations, allowing a wide variety of parameters and conditions to be examined. Simulations were carried out using LAMMPS (version 3 March 2020), ${ }^{58,59}$ and visualisation and analysis of simulation trajectories were conducted using OVITO. ${ }^{60}$

The GB potential ${ }^{61}$ is an anisotropic form of the widely used Lennard-Jones (LJ) pair potential, and captures the short-ranged excluded-volume repulsion and longer ranged van der Waals attraction between uncharged anisotropic molecules. The potential is characterised by parameters $\sigma$ and $\varepsilon$ that define the length and energy scales of the potential, respectively, parameters $v$ and $\mu$ that tune the shape of the potential, and, for uniaxial particles, the short and long principal diameters, $\sigma_{\mathrm{F}}$ and $\sigma_{\mathrm{S}}$, and relative well depths, $\varepsilon_{\mathrm{F}}$ and $\varepsilon_{\mathrm{S}}$, along the corresponding axes (the subscripts "F" and "S" denote "face" and "side", respectively). The anisotropy of the uniaxial particles is described by the shape anisotropy parameter $\kappa=\sigma_{\mathrm{F}} / \sigma_{\mathrm{S}}$ and the interaction anisotropy parameter $\kappa^{\prime}=\varepsilon_{\mathrm{S}} / \varepsilon_{\mathrm{F}}$. A full description of the GB potential and parameters is given in the ESI, section S1.

In all simulations, we have used $v=1$ and $\mu=2$, as is common for the GB potential, in line with its original parameterisation ${ }^{61}$ and previous parameterisations of OSC systems, ${ }^{62}$ and and have set $\varepsilon_{\mathrm{S}}=1$ and $\sigma_{\mathrm{F}}=1.03 \sigma$. A non-bonded interaction cutoff of $3 \sigma_{\mathrm{S}}$ was used for all systems. In order to match experimentally relevant systems, published GB parameters for biaxial models of several simple OSCs ${ }^{62}$ were used as a starting point to define the interactions in the systems studied here. To obtain a simpler uniaxial representation of each molecule, for which the end-end and side-side interactions and dimensions are equivalent, the published biaxial principal-diameter and well-depth parameters for end-end and side-side interactions were averaged. This typically resulted in changes to these parameters of less than
Table 1 Shape anisotropy parameter $\kappa$ and interaction anisotropy parameter $\kappa^{\prime}$ for uniaxial models of some typical organic semiconductors (OSCs) and benzene based on published parameters for biaxial models. ${ }^{62}$ The side-side parameters used to calculate the anisotropies in each uniaxial model were obtained as the average of the side-side and end-end parameters in the biaxial model, as described in the main text.

\begin{tabular}{c|cc}
\hline molecule & $\kappa$ & $\kappa^{\prime}$ \\
\hline porphine & 0.28 & 0.159 \\
pyrene & 0.34 & 0.164 \\
perylene & 0.36 & 0.190 \\
benzene & 0.47 & 0.270 \\
\hline
\end{tabular}

10 and $20 \%$, respectively, from their original published values. The resulting shape and interaction anisotropies of several small organic molecules are given in Table 1 and inform the values of $\kappa$ and $\kappa^{\prime}$ explored.

We have used LJ reduced units throughout this work, with energies, lengths, temperatures, pressures, and times given in units of $\varepsilon, \sigma, \varepsilon / k_{\mathrm{B}}, \varepsilon / \sigma^{3}$, and $\tau \equiv \sqrt{m_{0} \sigma^{2} / \varepsilon}$, where $m_{0}$ is the unit of mass and $k_{\mathrm{B}}$ is the Boltzmann constant. We note that setting $\varepsilon=1.8 \mathrm{kcal} / \mathrm{mol}$ and $\sigma=3.2 \AA$ gives behaviour consistent with the experimental behaviour of perylene, i.e. a density of $1.1 \mathrm{~g} / \mathrm{cm}^{3}$ at $560 \mathrm{~K}$ and $1 \mathrm{~atm}$ for a system with $\kappa$ and $\kappa^{\prime}$ approximately those of perylene in Table $1,{ }^{62}$ and so the parameter range studied should be representative of the behaviour of a variety of OSCs given this choice of $\varepsilon$ and $\sigma$.

To mimic the scaling of molecular mass with molecular size typical of OSCs, the particle mass $m$ was scaled in proportion to the particle volume $v_{\mathrm{f}}=\pi \sigma_{\mathrm{F}} \sigma_{\mathrm{S}}^{2} / 6$ (calculated as the volume for an ellipsoid with principal diameters $\sigma_{\mathrm{F}}, \sigma_{\mathrm{S}}$, and $\sigma_{\mathrm{S}}$ ) to give a constant mass density within the particle. Although approximate, this behaviour is reasonably representative of real systems, for which the mass density within the particle generally varies between 1.4 and $1.7 \mathrm{~g} / \mathrm{mol} / \AA^{3}$ when the particle volume is calculated from published biaxial GB parameters ${ }^{62}$ (ESI Table S3). To facilitate comparison between systems with different sized particles, we have analysed results in terms of the volume fraction, $\phi$, rather than the number density, $\rho_{\mathrm{b}}$, in the bulk fluid. The two are related as $\phi=\rho_{\mathrm{b}} / v_{\mathrm{f}}$.

To encompass the region of parameter space in Table 1 for typical OSCs, values of $\kappa$ of $0.30,0.35,0.40,0.45$, and 0.50 , and $\kappa^{\prime}$ of $0.15,0.20,0.25$, and 0.30 were used. Additional $\kappa^{\prime}$ values of 0.50 and 0.70 were examined for certain systems to access points with $\kappa^{\prime}>\kappa$, as interfacial behaviour has previously been predicted to exhibit a transition around $\kappa / \kappa^{\prime}=1$ in some circumstances. ${ }^{36}$ Several systems with $\kappa^{\prime}=1.2$ were also studied to examine the behaviour when side-side interactions are stronger than faceface ones. While side-side interactions that are stronger than the face-face ones are not typical of OSCs-like molecules, they can potentially be accessed through, for example, functionalisation of the aromatic core and may present an interesting means of tuning interfacial orientation. The systems with $\kappa^{\prime}>1$ in this work had weaker face-face interactions than the systems with $\kappa^{\prime}<1$ in order to maintain an isotropic bulk fluid as the side-side interactions were increased, meaning face-face interactions were weaker than would be the case for a typical OSC. As the alignment 
at both solid and vapour interfaces is expected to depend on temperature, due to competition between excluded-volume entropic effects and attractive intermolecular interactions, ${ }^{42}$ several temperatures between 0.56 and $0.84 \varepsilon / k_{\mathrm{B}}$ were studied, corresponding to a temperature range of $507-761 \mathrm{~K}$ for the representative OSC systems described above, encompassing a range of temperatures where the OSCs-type molecules that are represented by these GB parameters are liquid. A full list of the specific systems studied is given in the ESI, Table S6.

Simulations were conducted with the fluid confined in the $z$ direction between two fixed interfaces parallel to the $(x, y)$-plane, as illustrated in Fig. 2a: a particle-based solid surface at the bottom and a perfectly flat wall at the top that interacts with the center of each fluid particle with a repulsive harmonic potential,

$$
u_{\mathrm{w}}(z)= \begin{cases}\varepsilon_{\mathrm{W}}\left(z-z_{\mathrm{W}}\right)^{2}, & z>z_{\mathrm{W}} \\ 0, & \text { otherwise }\end{cases}
$$

where $z_{\mathrm{W}}$ is the wall position, $\varepsilon_{\mathrm{W}}$ describes the strength of the wall-particle interaction, and $z$ is the vertical position of the centre of the fluid particle. This repulsive wall was implemented at the top surface of the fluid to maintain an approximately constant average fluid density between systems with particles of the same shape and to prevent evaporation. Similar to a vapour interface, this wall constrained only the vertical position of the centre of a fluid particle and placed no constraints on the position of the particle surface defined by the repulsive part of the GB potential. We will refer to this vapour-like interface as the "vapour interface" in what follows, and note that it is comparable to the penetrable walls used in previous studies of interface ordering of anisotropic particles. ${ }^{56,57}$ In contrast, no part of a fluid particle could penetrate the solid surface.

The solid substrate was modelled as a single layer of either atomistic graphene or silicon, positioned with atoms centred at $z=0$. These substrates have been shown experimentally to interact strongly (graphene) or weakly (silicon) with OSCs and to give rise to face-on and side-on orientations, respectively, of the common OSC polymer poly(3-hexylthiophene) (P3HT). ${ }^{51}$ Parameters (non-bonded interactions and bond length) for graphene were taken from the OPLS-AA force field ${ }^{63}$ for aromatic carbons $\left(\varepsilon_{\mathrm{LJ}}=\right.$ $0.039 \varepsilon, \sigma_{\mathrm{LJ}}=1.11 \sigma$, bond length $=0.44 \sigma$, for $\varepsilon=1.8 \mathrm{kcal} / \mathrm{mol}$ and $\sigma=3.2 \AA$ ) and atoms positioned in the hexagonal lattice of graphene with separation taken from the OPLS-AA force field as the bond length for aromatic carbons. ${ }^{63}$ OPLS parameters were also used for silicon ${ }^{64}\left(\varepsilon_{\mathrm{LJ}}=0.056 \varepsilon, \sigma_{\mathrm{LJ}}=1.70 \sigma\right)$ and atoms positioned to give the fcc(001) plane of silicon's diamond cubic lattice (lattice spacing of $1.69 \sigma$ ). ${ }^{65}$ Although $\varepsilon_{\mathrm{LJ}}$ is larger for silicon, the larger lattice spacing means that the overall attraction of a single GB fluid particle to the substrate is weaker for silicon than graphene. An additional substrate with the same structure as graphene but with interactions that were twice as strong $\left(\varepsilon_{\mathrm{LJ}}=0.078 \varepsilon, \sigma_{\mathrm{LJ}}=1.11 \sigma\right)$ was also examined, which we will call the "strong" substrate. The structures of these substrates are shown in the ESI, Fig. S1. The mixing rules for combining GB parameters of two dissimilar particles, ${ }^{62}$ defined by eqns (S3)-(S8) of the ESI, were used to model the fluid-substrate interactions so (a)

(b)

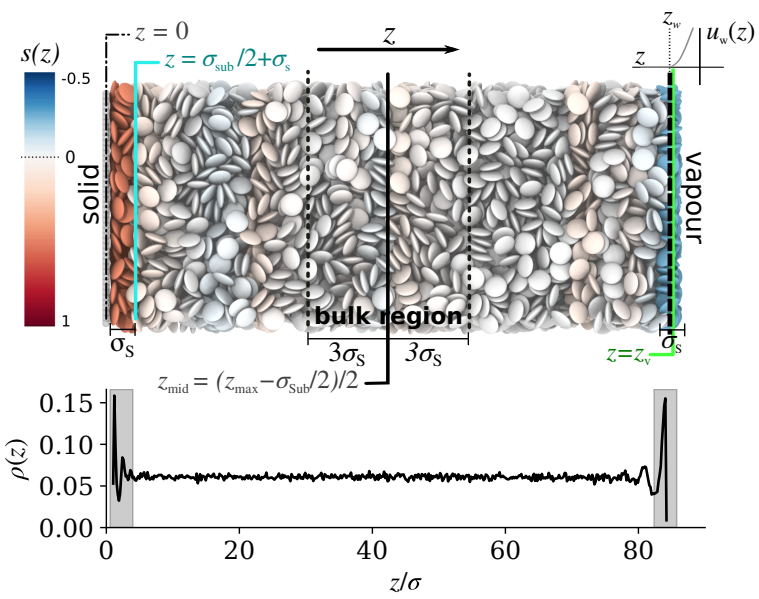

(c)

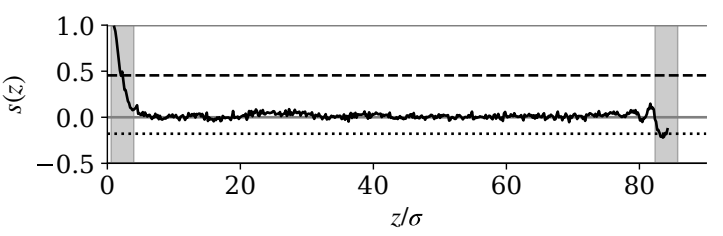

Fig. 2 (a) Example of the system setup, with Gay-Berne (GB) fluid particles confined between two surfaces: the impenetrable solid surface consisted of a single layer of Lennard-Jones (LJ) particles, centered at $z=0$ (black dash-dot line), while a repulsive harmonic potential acted on the centre of each fluid particle at the penetrable vapour-like interface (dashed black line) to prevent evaporation. The plot at the top right is representative of the form of this potential. The bulk region was defined as being within $\pm 3 \sigma_{\mathrm{S}}$ of the $z$ coordinate of the centre of the box ( $z_{\text {mid }}$, black solid line). $z_{\max }$ is the maximum $z$ coordinate of any particle during the simulation, $\sigma_{\text {sub }}$ the diameter of a substrate particle, and dotted black lines indicate the bounds of the bulk region. This region was sufficiently far from both interfaces that, except for a couple of the systems that formed anisotropic phases, the fluid had uniform density here. The position of the vapour interface, $z_{\mathrm{v}}$ (green line), was defined as the $z$-coordinate where the average density was equal to half the average bulk value. The average molecular orientation at the vapour interface, $s_{\text {vap }}$, was calculated as the average of $s(z)$ from eqn (2) over particles whose centres were at $z$ coordinates within $z_{\mathrm{v}} \pm \sigma_{\mathrm{S}} / 2$ (region indicated in figure). The average molecular orientation at the solid interface, $s_{\text {sub }}$, was calculated by averaging $s(z)$ over fluid particles whose centres were within $\sigma_{\mathrm{S}}$ of the surface of the substrate (i.e. at $z<\sigma_{\mathrm{sub}} / 2+\sigma_{\mathrm{S}}$, cyan line). These definitions selected, to a good approximation, the particles within the first fluid layer at either interface. Particles are coloured by the orientational order parameter $s(z)$ (which is -0.5 and +1 , respectively, for fully side-on and face-on orientations), calculated for layers of width $z=$ $\sigma_{\mathrm{S}}$ for a snapshot of the sample system $\left(\kappa=0.3, \kappa^{\prime}=0.3, T^{*} \equiv k_{\mathrm{B}} T / \varepsilon=\right.$ 0.73 , substrate $=$ graphene). (b) Fluid density $\rho(z)$ and (c) orientational order parameter $s(z)$ profiles calculated over the entire simulation of this system. The shaded areas correspond to the interfacial fluid regions at the solid or vapour interface. Dashed and dotted horizontal lines in (c) correspond to the values of $s_{\text {sub }}$ and $s_{\text {vap }}$ respectively. Additional $\rho(z)$ and $\phi(z)$ profiles can be found in the ESI, section S5. 
as to maintain the correct shape dependence of the interactions. The interaction range $\sigma$ in eqn (S2) of the ESI was replaced by the arithmetic mean, $\left(\sigma+\sigma_{\mathrm{LJ}}\right) / 2$, of the fluid and substrate interaction ranges for the fluid-substrate interactions. The substrate parameters, and the influence of the type of substrate on the orientation at both interfaces are given in full in the ESI (Table S4, Fig. S2).

To give comparable steepness for the repulsive potential at the vapour interface to the interaction with the solid substrate, the value of $\varepsilon_{\mathrm{w}}$ was determined by fitting eqn (1) to the repulsive part of the potential energy versus $z$ of a single fluid particle representative of perylene $\left(\kappa=0.36, \kappa^{\prime}=0.19\right)$ interacting with the solid substrate in the face-on orientation for the region where the potential energy was $<2 k_{\mathrm{B}} T$ at $T=0.62 \varepsilon / k_{\mathrm{B}}$. This gave a value of $\varepsilon_{\mathrm{W}}=17.8 \varepsilon$ for the graphene substrate and $\varepsilon_{\mathrm{W}}=8.9 \varepsilon$ for the silicon substrate. The same values for each substrate were used for both the attractive and repulsive surfaces, and the graphene value also used for the "strong" substrate, as the strength of the wall potential did not significantly affect the behaviour at the vapour interface (ESI Fig. S2).

All simulations were conducted at constant volume and temperature with the positions of the substrate particles fixed. Each system consisted of 6000 fluid particles and 4680 (graphene) or 800 (silicon) substrate particles. The simulation timestep was $0.012 \tau$, which corresponds to 5 fs if the unit of mass $m_{0}$ is taken to be the molecular mass of perylene. Temperature was controlled with a Nosé-Hoover thermostat ${ }^{66,67}$ and set to values of $0.56,0.62,0.73$, or $0.84 \varepsilon / k_{\mathrm{B}}$. Most systems were constrained to a constant overall volume fraction $\phi_{\mathrm{av}}$ (that is, the volume fraction calculated from the number density of all particles between $z=\sigma_{\text {sub }} / 2$, the surface of the substrate, and $z=z_{\mathrm{w}}$, the position of the harmonic wall) of 0.39 , corresponding to a real density on the order of $1.1 \mathrm{~g} / \mathrm{cm}^{3}$ if $\varepsilon=1.8 \mathrm{kcal} / \mathrm{mol}$ and $\sigma=3.2 \AA$. The $z$ coordinate of the harmonic wall, chosen to achieve the desired overall volume fraction for the different values of $\kappa$, are given in the ESI Table S1. Additional systems were examined at lower (0.28) and higher (0.49) $\phi_{\mathrm{av}}$ values to determine the dependence (if any) of interfacial orientation on the system density. The pressure in each system was measured as the normal force per unit area acting on the solid substrate. The average pressure at equilibrium was generally $<200 \varepsilon / \sigma^{3}$, but was up to $1200 \varepsilon / \sigma^{3}$ for $\phi_{\mathrm{av}}=0.49$.

To initialise the simulations, fluid particles were packed with random positions and orientations into a large box (periodic in $x, y$, and $z$, with $x$ and $y$ dimensions $10 \times$ the target values and $z$ dimension equal to the target value). For systems to be simulated with the graphene substrate, the target $x$ and $y$ dimensions were $34.48 \sigma$ and $34.125 \sigma$, respectively, to enable periodic packing of the substrate particles; for the silicon substrate, the final dimensions were $x=y=33.94 \sigma$ for the same reason. The size in the $z$ dimension was used to control the volume fraction of fluid particles and so depended on fluid particle size; the values for various systems can be found in the ESI Table S1. A soft potential $U_{\text {soft }}(r)=A\left[1+\cos \left(\frac{\pi r}{r_{\mathrm{c}}}\right)\right]$ for $r<r_{\mathrm{c}}$, where $r_{\mathrm{c}}$ is is a cutoff distance and $A$ an energy pre-factor that varies the "hardness" of the potential, was applied to remove particle overlaps $\left(r_{\mathrm{c}}=5 \sigma, A\right.$ increased linearly from 0 to $16.7 \varepsilon$ over 20,000 timesteps). Interactions were changed from the soft potential to the GB potential, and the box dimensions shrunk linearly over 50,000 timesteps at a higher temperature of $T=0.88 \varepsilon / k_{\mathrm{B}}$ to give the final dimensions outlined above. To initialise the system in an isotropic phase, the fluid particles were then allowed to equilibrate in the bulk system at $T=0.88 \varepsilon / k_{\mathrm{B}}$ for another 50,000 time steps. The periodic boundary conditions in the $z$ direction were then removed, and the box extended in the $z$ direction to give a region of vacuum above the bulk fluid. The solid substrate was introduced below the fluid, and the harmonic wall at the vapour interface was positioned at the $z$ coordinate required to give the desired overall volume fraction (ESI Table S1). The energy of the system was then minimised to remove any overlaps between fluid and interfaces. The system was simulated for a further 510,000 timesteps at the specified temperature, with the equilibration and correlation time for the orientation of the top and bottom fluid layers determined using pymbar's timeseries module, ${ }^{68,69}$ which estimates the equilibration time as the time that maximises the number of uncorrelated samples (see ref. 70 for details). Only the data after the equilibration time was used for further analysis. Although a number of systems were simulated that were anisotropic in the bulk (typically for the most anisotropic shapes and interactions; see ESI Table S6 for a list of systems that formed anisotropic bulk phases), the slow dynamics in these systems did not allow equilibrium properties to be measured reliably over the time scales that were simulated. Results are therefore only reported in the main paper for systems with an isotropic bulk fluid phase.

\subsection{Purely repulsive particle simulations}

As their interactions have, to a good approximation, no energetic component, purely repulsive particles were used to determine the contribution of entropy to molecular alignment at the solid and vapour interfaces. For these purely repulsive particles, the shape parameters described above (with $\kappa=0.30,0.35,0.40,0.45,0.50$ ) were used, and $\kappa^{\prime}$ was fixed at $0.2\left(\varepsilon_{\mathrm{S}}=1, \varepsilon_{\mathrm{F}}=5\right)$. The GB potential was cut-off and shifted to 0 at the minimum for each orientation to obtain a purely repulsive potential, ${ }^{71}$ analogous to the Weeks-Chandler-Andersen (WCA) truncation of the LJ potential. ${ }^{72}$ Likewise, a cut-off and shifted form of the fluid-substrate interactions, using the mixing rules for combining GB parameters of two dissimilar particles ${ }^{62}$ discussed previously, was used for the interactions with the solid substrate. These simulations were conducted at $T=0.62 \varepsilon / k_{\mathrm{B}}$ at overall volume fractions between 0.1 and 0.5 using either the silicon or graphene substrate. Although these systems are expected to be athermal, simulations were also carried out for the silicon substrate at $T=0.8 \varepsilon / k_{\mathrm{B}}$ to verify this fact. A full list of the systems studied is given in ESI Table S5. Data for the different substrates and temperatures was combined to determine the scaling of interface orientation with system parameters (see ESI, section S4).

\section{Results and discussion}

We have examined the orientation of anisotropic particles with both attractive and repulsive interactions at solid and vapour in- 
terfaces. The average molecular orientation as a function of the coordinate, $z$, perpendicular to the interface was quantified by the orientational order parameter

$$
s(z)=\left\langle P_{2}(\cos \theta)\right\rangle_{z}=\frac{3\left\langle\cos ^{2} \theta\right\rangle_{z}-1}{2},
$$

where $\theta$ is the angle between the short (unique) particle axis and the $z$ axis, and the average $\langle\cdots\rangle_{z}$ is over all fluid particles whose centres were in the histogram bin centred at position $z$. A value of 1 indicates fully face-on alignment, and $-\frac{1}{2}$ indicates fully sideon. For isotropic orientations, $s(z)=0$. Throughout this work, the terms face-on and side-on will be used to describe any orientation that is either predominantly face-on $(s(z)>0)$ or side-on $(s(z)<$ 0 ), not just the fully aligned extremes. Representative density and orientation profiles are shown in Fig. 2, and selected additional profiles are given in the ESI, section S5.

For a bulk nematic GB fluid, the orientation at the vapour interface has previously been shown to scale with the ratio of the shape and interaction anisotropy parameters, $\kappa / \kappa^{\prime}$, with a faceon orientation observed for $\kappa / \kappa^{\prime}<1$, and an side-on orientation otherwise. ${ }^{36}$ For the systems studied in this work, in which the bulk phase is isotropic rather than nematic and in which solid interfaces are also considered, Fig. 3 shows that $\kappa / \kappa^{\prime}$ is not a good predictor of orientation at either the solid or vapour interface. At the vapour interface, the orientation does not appear to depend on $\kappa / \kappa^{\prime}$ for $\kappa / \kappa^{\prime}>1$, although there seems to be a transition to very marginally face-on orientations at $\kappa / \kappa^{\prime}<1$, as predicted previously for bulk nematic fluids. ${ }^{36}$ The systems that display a slightly face-on orientation are those with the most isotropic interactions studied here $\left(\kappa^{\prime}=0.7\right)$, resulting in very slight orientation preference, while those with the more face-on orientation are where side-side interactions are stronger than face-face $\left(\kappa^{\prime}=1.2\right)$. Stronger alignment could be achieved by decreasing $\kappa^{\prime}$ (increasing interaction anisotropy), but the corresponding increase in the shape anisotropy $\kappa$ required to maintain $\kappa / \kappa^{\prime}<1$, coupled with the more anisotropic interactions, would likely result in systems that are anisotropic in the bulk. At the solid interface, there is a weak trend towards a more face-on orientation as $\kappa / \kappa^{\prime}$ decreases within each set of substrate/temperature conditions, but there is a strong dependence on temperature and $\kappa / \kappa^{\prime}=1$ does not correspond to the transition from side-on to face-on orientation. This dependence on temperature points to a significant entropic contribution, which is only accounted for implicitly in the $\kappa / \kappa^{\prime}$ parameter through the shape anisotropy $\kappa$.

Thus, we have considered an alternative metric for predicting the orientation at both solid and vapour interfaces based on an estimate of the free-energy difference between the face-on and sideon orientations, which explicitly accounts for the roles of temperature and fluid-substrate interactions. The overall free energy includes an entropic term, $\Delta \bar{S}$, described in section 3.1 and calculated based on the shape anisotropy, $\kappa$, and bulk volume fraction, $\phi$, of the system, and an energetic term, $\Delta \bar{U}$, described in section 3.2 and based on the interactions between nearest-neighbour particles. The free energy difference per interface particle be- (a)

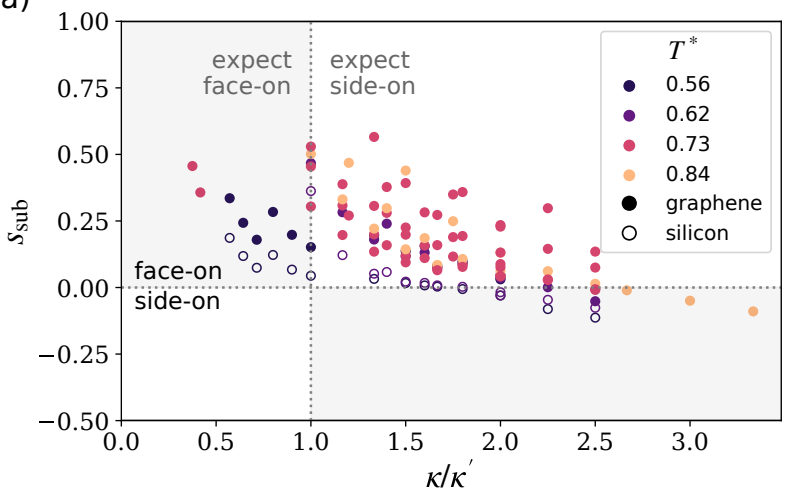

(b)

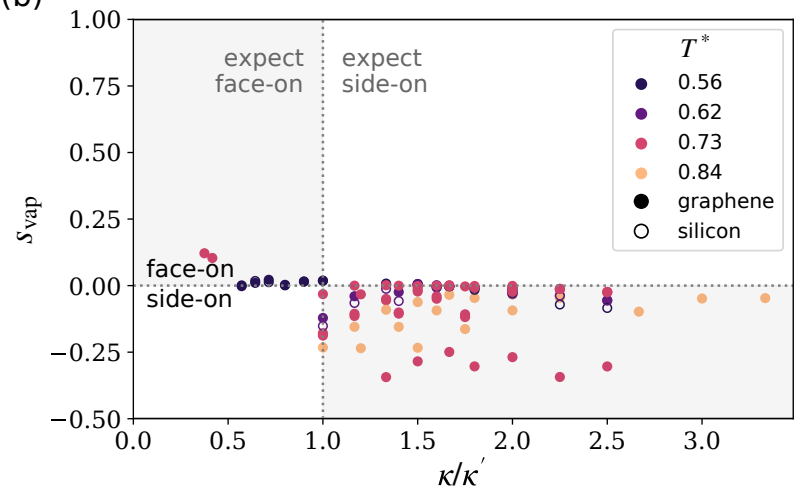

Fig. 3 Interface orientational order parameter, $s$, versus shapeanisotropy:interaction-anisotropy ratio, $\kappa / \kappa^{\prime}$, at the (a) solid and (b) vapour interfaces for graphene-structure (graphene or "strong"; filled symbols) and silicon (unfilled symbols) substrates and various reduced temperatures, $T^{*} \equiv k_{\mathrm{B}} T / \varepsilon$. 
tween face-on and side-on orientations is calculated as

$$
\Delta \bar{F}=\Delta \bar{U}-T \Delta \bar{S} .
$$

where overbars indicate per-particle quantities. A predominantly face-on orientation is predicted for $\Delta \bar{F}<0$, and side-on for $\Delta \bar{F}>$ 0 .

\subsection{Entropic component}

Although a number of theories exist to quantify the entropy difference between face-on and side-on interface configurations, ${ }^{33,37,40-42}$ a simple, accurate, analytical expression for the entropy as a function of system parameters is lacking, and a nematic bulk phase is often assumed. ${ }^{37}$ Instead, we have taken a semi-empirical approach, in which the entropy difference is measured in a comparatively small number of MD simulations of purely repulsive particles, to obtain a universal scaling relationship at the solid and vapour interfaces.

Due to the absence of attractive interactions, any alignment of the purely repulsive particles at the solid or vapour interfaces can, to a good approximation, be attributed entirely to entropy. The Helmholtz free energy difference between two states of a system can be calculated as $\Delta F=\Delta U-T \Delta S$, which in the absence of an energetic contribution $(\Delta U=0)$ gives $\Delta F=-T \Delta S$. The entropy difference per interface particle between states of a system in the NVT ensemble with exclusively face-on and side-on orientations at an interface can then be estimated as

$$
\Delta \bar{S}=-\frac{\Delta \bar{F}}{T}=k_{\mathrm{B}} \ln \left(\frac{P_{\mathrm{F}}}{P_{\mathrm{S}}}\right),
$$

where $P_{\mathrm{F}}$ and $P_{\mathrm{S}}$ are the probabilities of finding a fluid particle in the solid or vapour interfacial region (defined in Fig. 2) in the perfectly face-on $(\cos (\theta)=1)$ and side-on $(\cos (\theta)=0)$ orientations, respectively. Examples of the distribution of $\cos (\theta)$ in the interfacial regions are given in the ESI, Fig S6.

The orientation at the solid interface was found always to be predominantly face-on, while a predominantly side-on orientation was exclusively found at the vapour interface (Fig. 4). In both cases, the degree of alignment was found to depend systematically on the volume fraction of the bulk fluid and the shape anisotropy of the fluid particles, with higher densities and shape anisotropies giving a greater degree of alignment. It has previously been predicted that a variety of hard anisotropic particles align with their long axis parallel to a solid substrate, ${ }^{37-41}$ as this alignment maximises the free volume and hence is the entropically favoured orientation. ${ }^{37,41}$ The opposite argument can be applied at the vapour interface, where the particles are able to extend over the interface, increasing the free volume when their long axis is perpendicular to the interface. ${ }^{33}$ Studies of hard ellipsoids at penetrable walls also show this behaviour. ${ }^{56,57}$ Thus, for oblate particles, a face-on orientation at the solid interface and a side-on orientation at the vapour interfaces are predicted to be entropically favoured, which is qualitatively consistent with the results presented here.

The entropy difference between face-on and side-on interface configurations was found to have a quasi-universal power-law
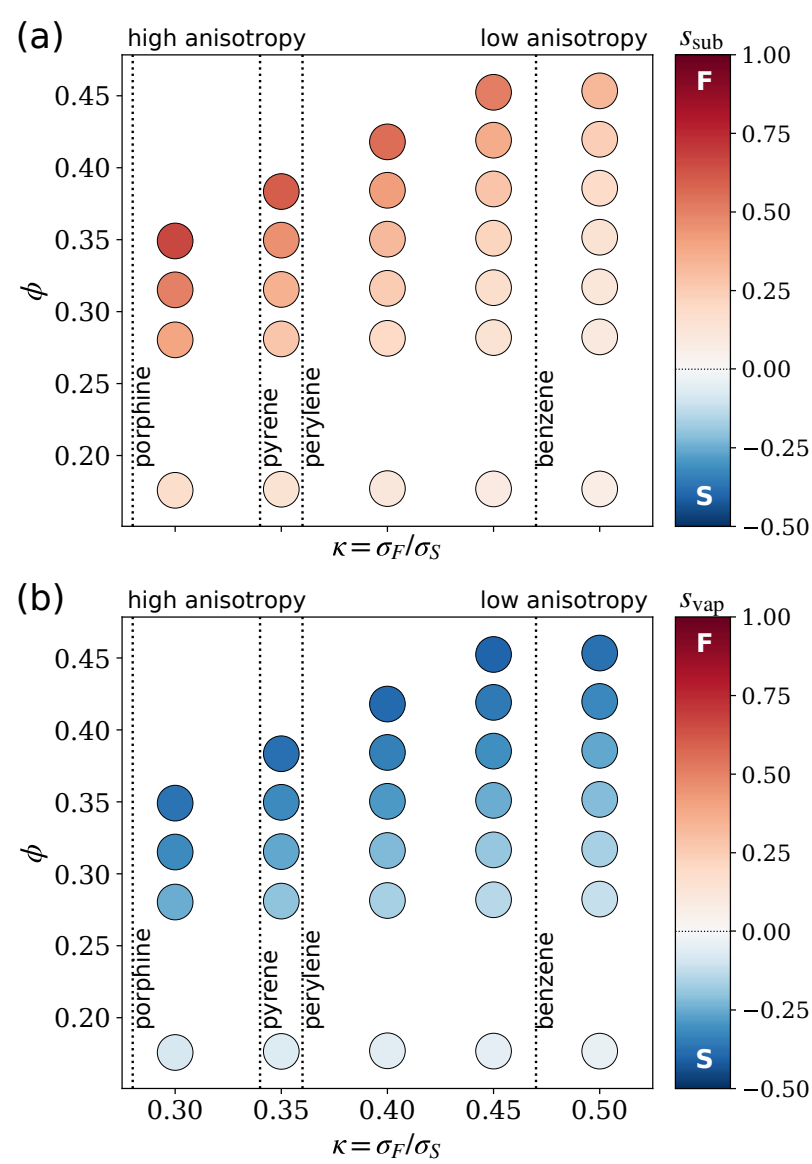

Fig. 4 Orientational order parameter of purely repulsive fluid particles at (a) a purely repulsive graphene-like substrate and (b) the vapour interface as a function of shape anisotropy and bulk fluid volume fraction at $T=$ $0.62 \varepsilon / k_{\mathrm{B}}$. The colour of each point indicates the value of the order parameter at each interface. The shape anisotropy of various OSCs is indicated for reference. The labels ' $F$ ' and ' $S$ ' in the colour bar indicate face-on and side-on orientations, respectively. The orientational order parameter value for this and the other substrates and temperatures can be found in the ESI, Table S5. (The missing points at low $\kappa$ and high $\phi$ formed anisotropic bulk phases and could not be equilibrated on the simulation time scale, and so are not included.) 
dependence on $\phi / \kappa$ at the solid interface and on $\phi / \kappa^{1 / 2}$ at the vapour interface, where $\phi$ is the bulk fluid volume fraction and $\kappa$ is the shape anisotropy, as shown in Fig. 5, although there is some scatter in the data, particularly for the solid interface. The scaling relationships that best fit the data in Fig. 5 were

$$
\Delta \bar{S}_{\text {sub }}=2.89 k_{\mathrm{B}}\left(\frac{\phi}{\kappa}\right)^{2.51}
$$

at the solid interface and

$$
\Delta \bar{S}_{\text {vap }}=-26.34 k_{\mathrm{B}}\left(\frac{\phi}{\kappa^{1 / 2}}\right)^{3.53}
$$

at the vapour, where a negative value favours a predominantly side-on orientation and positive favours face-on.

While the scaling at the vapour interface with $\phi / \kappa^{1 / 2}$ is the same for both substrates, the relationship between the entropy difference and $\phi / \kappa$ for the silicon substrate is not as straightforward as that for the graphene substrate (Fig. 5a). Due to the spacing of the substrate particles (ESI Fig. S1), the fluid-substrate potential has more significant lateral corrugations at the silicon interface compared with graphene. This induces a greater dependence of the potential on the in-plane $(x, y)$ coordinates compared the graphene, which is much closer to an "ideal" planar solid surface for which the potential depends only on the $z$ coordinate. Despite the additional variability, the overall scaling behaviour follows the same trend at both substrates, and the fit to all the data was used.

Overall, if entropy is the only contributing factor and the system is isotropic in the bulk region, the orientation preference at the solid interface is expected to be face-on, and side-on at the vapour interface, consistent with previously published theories and calculations. ${ }^{33-42,56,57}$ Increasing the effect of entropy, such as by using higher temperatures, more anisotropic particles, or higher pressures (to increase density), could be used to enhance this alignment if desired at either interface.

\subsection{Energetic component}

The alignment of particles with attractive interactions at the solid interface is complicated by attractive interactions of the fluid with the substrate and within the fluid itself. Qualitatively, the faceon orientation should be energetically favoured when the facesubstrate interactions are strong (although this will also depend on the relative strength of the fluid face-face, side-side, and sidesubstrate interactions), and the side-on orientation should be energetically favoured when the fluid particle's face-face interactions are stronger than both their side-side and face-substrate interactions. Assuming that the fluid is isotropic everywhere except for the layer adjacent to the interface, and that a fully face-on particle interacts with six nearest neighbours through side-side interactions (hcp packing) while a side-on particle has two faceface interactions and two side-side interactions (cubic packing with inequivalent lattice spacing) with its nearest neighbours (as found in simulations in which the interface layer was close to fully aligned, as shown in ESI Fig. S7), the difference in energy per interfacial fluid particle between a completely face-on orientated (a)

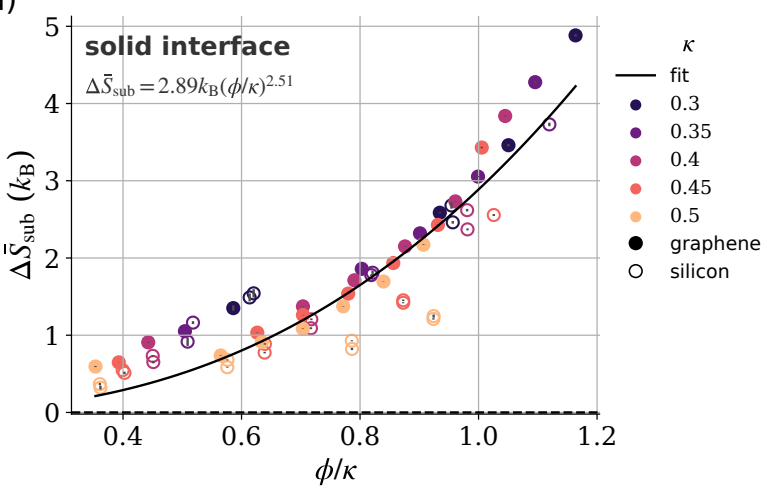

(b)

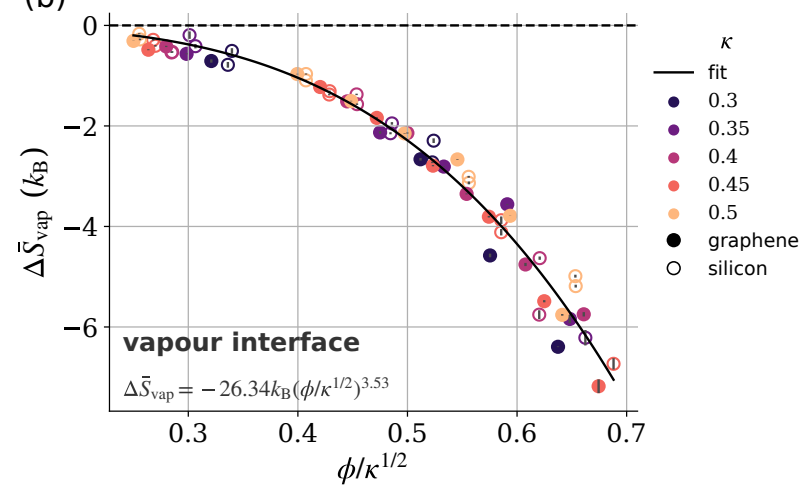

Fig. 5 Entropy difference per particle between face-on and side-on orientations as a function of $\phi / \kappa^{n}$ at the (a) solid $(n=1)$ and (b) vapour $(n=1 / 2)$ interfaces, where $\phi$ is the bulk volume fraction and $\kappa$ is the shape anisotropy of the fluid particles. The data here includes all sets of conditions for graphene- (filled symbols) and silicon-like (unfilled symbols) repulsive substrate at $T=0.62$ for $0.84 \varepsilon / k_{\mathrm{B}}$. The temperature does not significantly affect the scaling (ESI Fig. S3). Plots comparing the differences for different temperatures and substrate types are given in the $\mathrm{ESI}$, section $\mathrm{S} 4$ 
interfacial fluid layer and a completely side-on oriented one can be estimated to be

$$
\begin{aligned}
\Delta \bar{U} & =\bar{U}_{\mathrm{F}}-\bar{U}_{\mathrm{S}} \\
& =\left[\bar{U}_{\mathrm{Fs}}+6 \bar{U}_{\mathrm{Sf}} / 2\right]-\left[\bar{U}_{\mathrm{Ss}}+\left(2 \bar{U}_{\mathrm{Sf}}+2 \bar{U}_{\mathrm{Ff}}\right) / 2\right] \\
& =\bar{U}_{\mathrm{Fs}}-\bar{U}_{\mathrm{Ss}}+2 \bar{U}_{\mathrm{Sf}}-\bar{U}_{\mathrm{Ff}},
\end{aligned}
$$

where $\bar{U}_{\mathrm{F}}\left(\bar{U}_{\mathrm{S}}\right)$ is the interaction energy of a particle in the face-on (side-on) orientation with its nearest neighbours in the interfacial layer and with the substrate. (Note that the nearest-neighbour fluid interactions are halved in these equations because each such interaction contributes to the energy of two interfacial fluid particles.) $\bar{U}_{i j}$ is the interaction energy in the face-on/face-face orientation (for $i=\mathrm{F}$ ) or side-on/side-side orientation (for $i=\mathrm{S}$ ) with the substrate (for $j=\mathrm{s}$ ) or another fluid particle (for $j=\mathrm{f}$ ). For fluid-fluid interactions, the interaction energy in either the face-face or side-side orientation was taken as the minimum of the GB pair potential in the specified orientation. $\bar{U}_{\text {Fs }}$ and $\bar{U}_{\mathrm{Ss}}$ were calculated as the minimum in the interaction between a single GB ellipsoid and the substrate in the face-face and side-side orientations, respectively. As a particle-based substrate was used in this work rather than being perfectly flat, the energy minimum was averaged over 100 random positions on the substrate. Equation (7) also applies for the energy difference per fluid particle at the vapour interface, but with the fluid-substrate interactions set to zero, i.e. $\bar{U}_{\mathrm{Fs}}=\bar{U}_{\mathrm{Ss}}=0$.

\subsection{Predicting alignment at solid and vapour interfaces}

To predict the behaviour at both the solid and vapour interfaces, we have used the free energy described in eqn (3), with the entropic component determined from the bulk volume fraction and shape anisotropy of each system as described by eqns (5) and (6) for the solid and vapour interfaces, respectively, and with the energetic component determined from the nearest-neighbour interactions in the interfacial layer as described by eqn (7).

\subsubsection{Solid interface}

The average molecular orientation at the solid substrate, measured by the orientation order parameter $s_{\text {sub }}$, shows excellent universal scaling with the free energy difference defined by eqn (3) for the entire range of systems studied, covering temperatures from 0.56 to $0.84 \varepsilon / k_{\mathrm{B}}$, bulk volume fractions from 0.29 to 0.50 , strongly and weakly interacting substrates, and shape and interaction anisotropies corresponding to molecules as varied as benzene, perylene, and porphine (Fig. 6a; see ESI Table S6 for a full list of systems). Interestingly, although systems with a predominantly side-on orientation at the solid interface are observed, they are relatively rare, being obtained in less than 15\% of cases. These side-on systems generally correspond to situations for which the fluid particles have low shape anisotropy (high $\kappa$ ), the system is at low temperature, and to a lesser extent the fluid particles have high interaction anisotropy (low $\kappa^{\prime}$ ). The rarity of the side-on orientation points to a dominant entropic contribution in most systems studied.
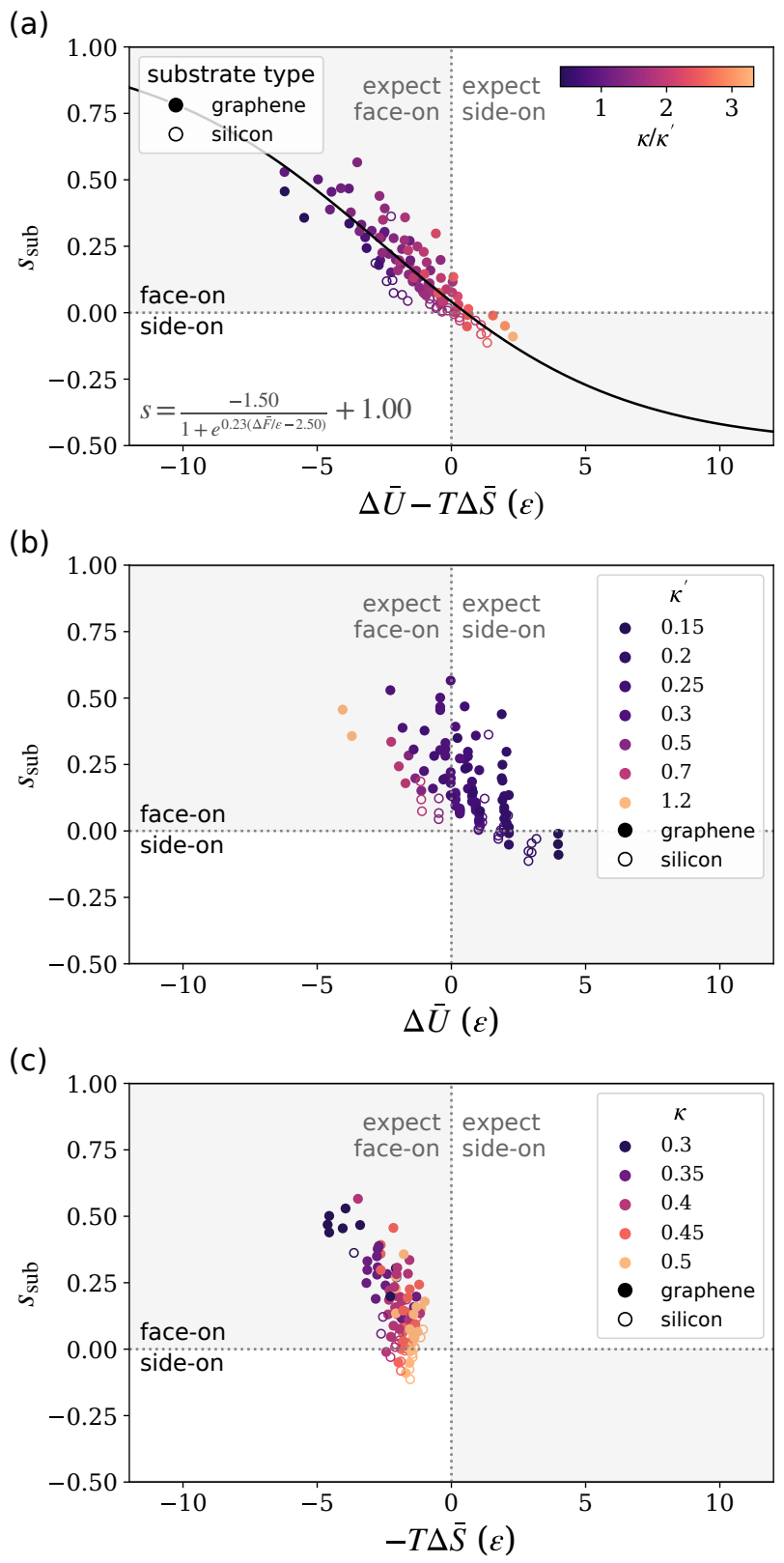

Fig. 6 Orientational order parameter $s_{\text {sub }}$ at the solid interface as a function of (a) overall, (b) energetic, and (c) entropic components of the interfacial free energy difference. A least-squares best fit to a logistic function, constrained to go to -0.5 and 1 at high and low $\Delta \bar{U}-T \Delta \bar{S}$ respectively, is shown in (a). The energetic component is coloured by the interaction anisotropy parameter, the entropy component by the shape anisotropy parameter, and the overall free energy by the ratio of the two parameters. Filled symbols correspond to the graphene-structure substrate (graphene or "strong") and unfilled symbols to the silicon substrate. Shaded grey regions highlight where the points should fall if obeying the predicted scaling with the free energy or free energy component. 
On separating the free energy into its energetic (Fig. 6b) and entropic (Fig. 6c) components, the reason for the preference for the face-on orientation at this interface becomes clear. Over the range of parameters studied, the energetic component varies from favouring the side-on orientation by values typically in the range of approximately $2 \varepsilon$, to the face-on orientation by a similar amount. Over the same range of parameters, the entropic component of the free energy always favours the face-on orientation by between 1 and $5 \varepsilon$, increasing in magnitude as the shape becomes more anisotropic. The combined result of these effects is an orientation that is only side-on under conditions where the entropic contribution is lowest (low temperature, low shape anisotropy) or the energetic contribution highest (highly anisotropic interactions). Although highly anisotropic interactions shift the energetic term significantly towards favouring the side-on orientation, high temperatures would be required in many cases to maintain an isotropic bulk fluid, which would increase the mangitude of the entropic term in the opposing direction, giving little change to the overall free energy. This separation of the free energy into its components also highlights that neither the energy nor the entropy is sufficient to completely explain the observed interface orientation, again emphasising the importance of both contributions for accurately predicting the orientation.

When examining the effect of the substrate on the interface orientation, a slight dependence is observed, with the observed orientation at the silicon substrate generally being shifted towards side-on relative to systems at the graphene-structured substrate (with either regular or strong interactions) for the same free energy difference (ESI Fig. S2). As described previously in the context of the purely repulsive systems, this slight substrate dependence is likely due to the looser packing of substrate particles in the silicon substrate compared with the graphene substrate. This increases the dependence of the fluid-substrate potential on the in-plane coordinates of the fluid particle at the silicon substrate, which behaves as a slighly penetrable surface, relative to the graphene substrate, which is much closer to an "ideal" smooth impenetrable solid substrate. No dependence on the strength of the fluid-substrate interactions was observed, however, with the results at the graphene and "strong" (same particle arrangement but double the interaction strength) substrates showing the same behaviour. This result indicates that patterning of the substrate may be an effective method to favour a more side-on alignment, as has been previously observed, 73 although further study would be required to fully understand this behaviour.

Although it appears difficult to significantly influence the interfacial orientation by tuning fluid-fluid interaction strength within the realm of reasonable OSC parameters while the bulk fluid remains in an isotropic liquid phase, the energetic component of the free energy difference does have a strong dependence on the strength of the fluid-substrate interactions. Switching from a strongly (e.g. graphite) to weakly (e.g. silica) interacting substrate has been shown experimentally $19,44,46,51-55$ and computationally ${ }^{45}$ to influence the orientation at the solid interface for a range of OSCs. Fig. 7 highlights the relative importance of the fluid-substrate interactions: the difference in energy on switching from a strongly to weakly interacting substrate (e.g. graphene

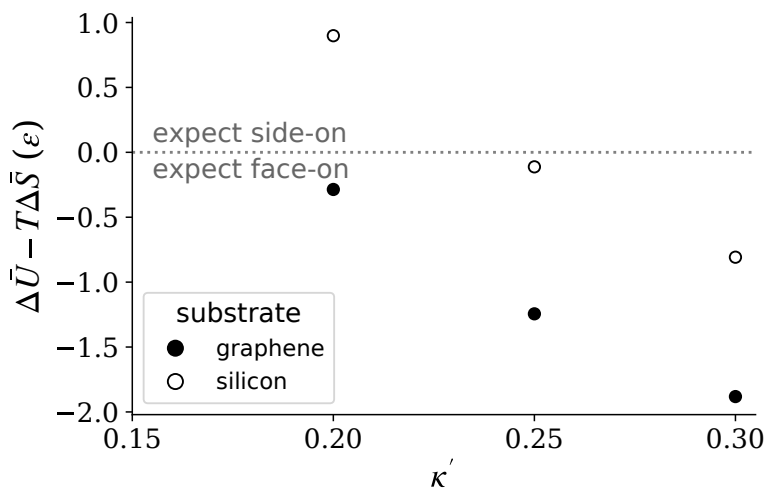

Fig. 7 Interface free energy difference as a function of interaction anisotropy parameter $\kappa^{\prime}$ at $T=0.62 \varepsilon / k_{\mathrm{B}}$ for graphene (filled symbols) and silicon (unfilled symbols) substrates.

to silicon) is comparable to changing the interaction anisotropy from values close to those representative of benzene to perylene (a significant structural change), and is sufficient to switch the free energy from favouring face-on to side-on orientation in some cases.

Although increasing the strength of the substrate-fluid interactions will increase both the side-substrate and face-substrate interaction strength, which make equal and opposite contributions to the energetic component of the interface free energy different in eqn (7), the face-substrate interactions are expected to dominate for most cases studied in this work due to two factors. First, the stronger face-face interaction compared with sideside interaction $\left(\kappa^{\prime}<1\right)$ typical of OSCs leads to the strength of face-substrate interactions increasing more rapidly than that of side-substrate interactions when the substrate-substrate interaction strength is increased isotropically, given the mixing rules for fluid-substrate interactions. Second, for oblate $(\kappa<1)$ particle shapes typical of OSCs (all the systems studied in this work), a face-on particle interacts with more substrate particles than a side-on particle, and so the strength of the interaction of a fluid particle with the whole substrate increases more rapidly for face-on particles than for side-on particles when the substratesubstrate interaction strength is increased isotropically, even if the fluid particles have no interaction anisotropy $\left(\kappa^{\prime}=1\right)$. Overall, while changing the fluid-fluid or fluid-substrate interactions are both plausible ways of influencing the energy, large structural changes would likely be required chemically to significantly change the fluid-fluid interaction anisotropy, which will likely also change the shape anisotropy and bulk phase behaviour of the fluid. It should generally be much simpler to change the nature of the solid substrate.

The fluid particles studied here are representative of small OSC molecules such as perylene that do not have any side-chains, but many OSCs feature alkyl side-chains designed to enhance their solubility. The different chemical nature of the backbone and side-chain (one being a conjugated $\pi$-system, the other an alkyl chain) means that changing the substrate to favour interactions with either one or the other should enhance either the face-on 
(strong backbone-substrate interactions) or side-on (strong sidechain-substrate interactions) orientations at the substrate interface. Although we do not consider any molecules with sidechains here, the impact of side-chains can be approximated in the coarse-grained modelling framework used in this work by considering their effect on the overall fluid-substrate interactions: a substrate that is more strongly attracted to the side-chains (particle side) will energetically favour the side-on orientation. This behaviour has been observed experimentally, for example though treatment of a substrate with a self-assembled monolayer of octadecyltrichlorosilane (OTS). ${ }^{74}$

\subsubsection{Vapour interface}

In contrast to alignment at the solid interface, which depends on the fluid-fluid and fluid-substrate interactions, the energetic driver for orientation at the vapour interface is simply expected to be related to the relative strength of the interactions for a face-on and side-on fluid particle with other fluid particles in the interfacial layer. Face-face interactions are maximised in the side-on orientation, so increasing the strength of these interactions is expected to promote the side-on orientation. Compared with the solid interface, the vapour interface does not show as clear universal scaling of the molecular orientation with the interface free energy parameter in eqn (3), but a strong trend with the free energy is still observed (Fig. 8a). Where $\kappa^{\prime}<1$, the orientation at this interface converges towards isotropic at the vapour interface as the free energy approaches zero, and becomes more aligned as the free energy magnitude increases. At high values of $\kappa^{\prime}$ (low interaction anisotropy), the energetic component (Fig. 8b) shifts towards very slightly favouring a face-on orientation, qualitatively consistent with previous predictions of ordering of bulk nematic fluids at the free interface. ${ }^{36}$

Several systems were also examined with $\kappa^{\prime}>1$, corresponding to stronger side-side than face-face interactions, as it is expected to promote the face-on orientation at the vapour interface. Even with reduced face-face interaction strength, bringing them outside the range of typical OSCs, most of these systems did not remain isotropic in the bulk. The two that did were the least anisotropic in shape and are the face-on outliers at $\Delta \bar{U}-T \Delta \bar{S} \approx 2 \varepsilon$ in Fig. 8a. While this result shows that it is possible to achieve a slight face-on orientation at the vapour interface for anisotropic particles while still maintaining an isotropic bulk phase, these points do not fall in the region where the simple free-energy predictor presented here predicts a face-on orientation.

The deviation from universal scaling at the vapour interface is likely due to several factors. Firstly, for a number of the systems, particularly those at high density, the pressure, measured as the normal force on the substrate, was relatively high - up to $1200 \varepsilon / \sigma^{3}$ in some cases. Examining the dependence of the relationship between $s_{\text {vap }}$ and the free energy on system pressure (ESI, Fig. S9b) shows more extreme side-on orientation (lower values of $s_{\text {vap }}$ ) at higher pressures for the same value of the free energy. While the orientation at the solid interface also shows a slight pressure dependence (ESI, Fig. S9a), the effect at the vapour interface is more pronounced. As the vapour interface is constrained by a repulsive harmonic wall, interacting with the
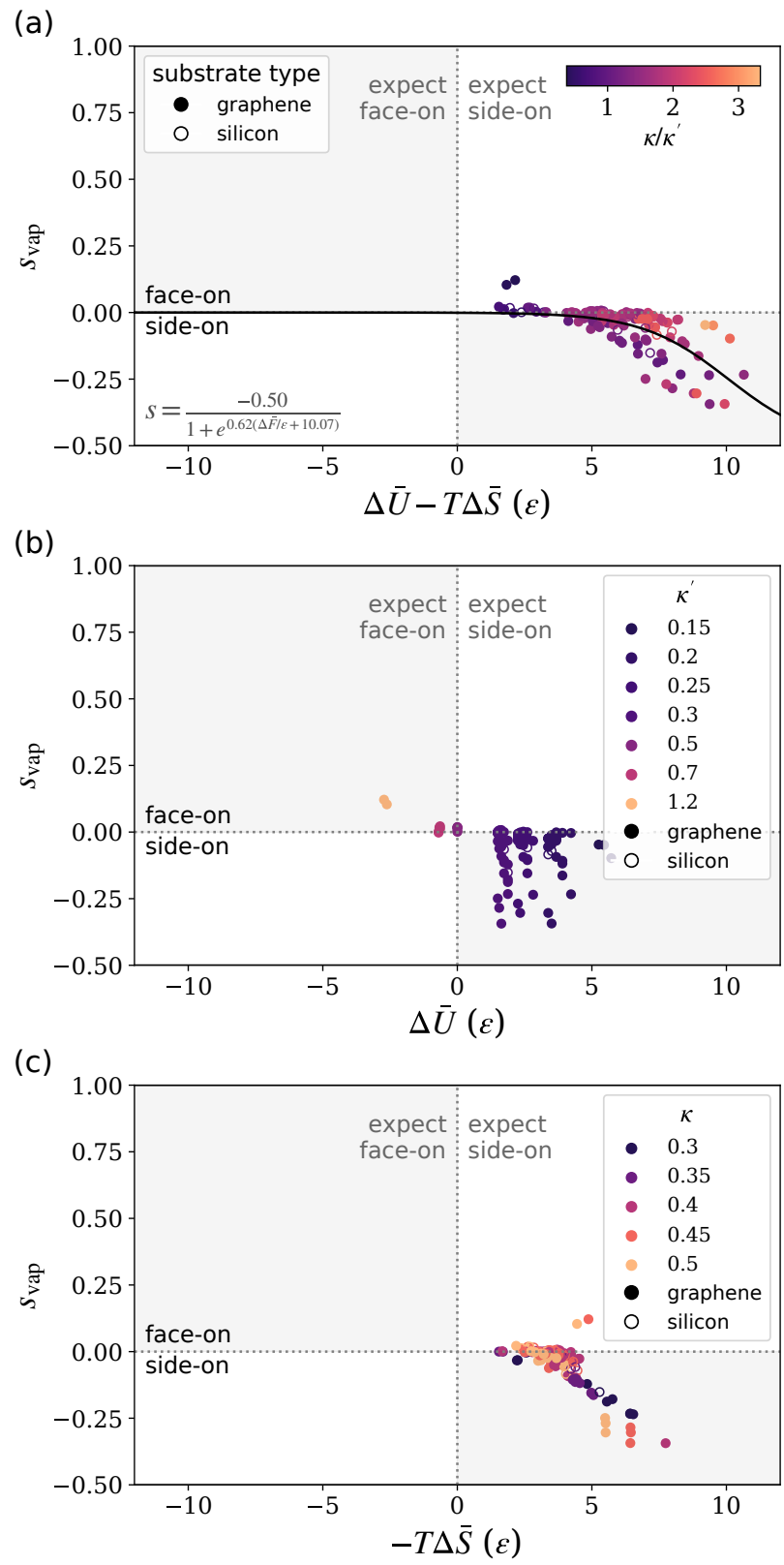

Fig. 8 Orientational order parameter $s_{\text {vap }}$ at the vapour interface as a function of the (a) overall, (b) energetic, and (c) entropic components of the interfacial free energy difference. A least-squares best fit to a logistic function, constrained to go to -0.5 and 0 at high and low $\Delta \bar{U}-T \Delta \bar{S}$ respectively is shown in (a). The energetic component is coloured by the interaction anisotropy parameter, the entropy component by the shape anisotropy parameter, and the overall free energy by the ratio of the two parameters. Filled symbols correspond to simulations with the graphenestructure substrate (graphene or "strong") and unfilled symbols to the silicon substrate. Shaded grey regions highlight where the points should fall if obeying the predicted scaling with the free energy or free energy component. 
centers of each fluid particle, high pressures correspond to a strong interaction between the fluid particles and this wall, which is likely not representative of a true vapour interface, where fluctuations are not artificially suppressed.

Secondly, as the energetic component of the interfacial free energy in eqn (7) is calculated assuming an idealised packing structure for a fully face-on and side-on interfacial layer, variations in the packing structure at the interface reduce the accuracy of the estimated free energy. The positions of fluid particles are less strongly constrained by the vapour interface than the solid substrate, and the side-on packing that predominates at the vapour interface appears to be less robust to disorder than the face-on packing that predominates at the solid interface, due to the tendency of nearest neighbours to be displaced parallel or angled slightly with respect to one another. Weaker ordering at the vapour interface results in molecular packings that deviate from the idealised structures, as shown in the ESI in Fig. S8. Similarly, the calculation of the energetic component of the free energy based solely on the fully face-on and fully side-on configurations means that the predictability of the model is likely to be poorer for systems with interfacial orientation that is closer to isotropic.

Despite these complications, the results indicate that it is difficult to obtain a face-on orientation under equilibrium conditions of isotropic bulk GB fluids for parameters representative of OSCs. For the systems studied here, the interaction energy favours the face-on orientation when the interaction strength is the most isotropic $\left(\kappa^{\prime}=0.7\right)$ of the values studied (Fig. 8b), though only marginally, and more significantly when $\kappa^{\prime}$ is increased to $>1$. In all cases, however, the entropic term (Fig. 8c) is sufficiently large that the overall free energy still predicts the side-on orientation. While significantly lowering the temperature would help to transition to the face-on orientation by reducing the entropic contribution, this is likely to result in a transition to a liquid-crystal or crystalline bulk phase. On the other hand, reducing the entropic component by reducing the shape anisotropy is likely to lead to a more isotropic interface orientation.

It has previously been shown that the orientation of similar GB particles at a nematic-vapour interface can be easily switched between face-on for $\kappa / \kappa^{\prime}<1$ to side-on $\kappa / \kappa^{\prime}>1,{ }^{36}$ whereas only a very slight preference for the face-on orientation was observed if $\kappa / \kappa^{\prime}<1$ for the isotropic-bulk systems studied here (Fig. 3b). The greater propensity for a nematic bulk fluid to give the face-on orientation at the vapour interface compared with an isotropic bulk fluid can be understood qualitatively in terms of differences between the interfacial energy of the face-on relative to the side-on orientation in the two cases. For the isotropic fluid, this energy is described by eqn (7), whereas for the nematic fluid, interactions with the adjacent anisotropic fluid layer must also be taken into account. Assuming that, compared with the isotropic fluid, each fluid particle in the interfacial layer of the nematic fluid interacts with an additional particle of the same orientation in the next fluid layer, the face-on orientation is predicted to be more energetically stabilised relative to the side-on orientation in the nematic fluid than in the isotropic fluid for the systems studied here with $\kappa^{\prime}<1$ and in ref 36 , for which the face-face interac- tions were always stronger than the side-side ones $\left(\kappa^{\prime}<1\right)$.

\subsection{Guidelines for controlling OSC interface orientation}

In all manner of OSC-based devices, orientation at interfaces has been correlated with device performance. ${ }^{9-18,20-23}$ Reliably controlling the orientation at these interfaces is therefore an important step towards designing high-performing molecules for commercial applications. Based on the results presented in the previous sections, we propose several general guidelines, which are supported by empirical evidence, for how the molecular structure, interface, and processing conditions may be tuned to shift towards the desired alignment at these interfaces.

Increased shaped anisotropy promotes a face-on orientation at the solid interface, and side-on at the vapour. As the shape anisotropy has a significant effect on the entropic component of the free energy described in eqn (3), it provides a simple handle for controlling orientation at both the solid and vapour interfaces. At the solid interface, increasing the shape anisotropy results in a shift towards a more face-on orientation (shifting left on the phase diagram presented in Fig. 6a), while the opposite behaviour is observed at the vapour interface. In both cases, this is due to an increase in the entropic driving force. Consistent with this prediction, a number of experimental studies have shown that backbone planarisation (induced by fluorination of the backbone, ${ }^{16,75,76}$ or extending the conjugation of the backbone, such as by adding aromatic components between the backbone and side-chains ${ }^{17}$, both of which can be associated with an increase in shape anisotropy), results in a more face-on orientation at the donor-acceptor interface in OPVs. It should be noted that both of these chemical modifications will also influence the energetics, so an interplay between changes in interaction strength and shape will control the orientation at the interfaces.

Stronger fluid face-face (or weaker side-side) interactions promote side-on orientation at both interfaces. Corresponding to a shift to the right on the plots in Figs. 6a,c and 8a,c, stronger face-face (or weaker side-side) interactions favour the side-on orientation in order to maximise these energetically more favourable interactions. As previously mentioned, this strategy is possibly the least effective approach for tuning the interface orientation as the energetic contribution to the interfacial free energy difference is close to zero for the interaction anisotropies studied here. Additionally, methods to tune the interaction anisotropy, such as introducing longer/bulkier side-chains that may block the face of the molecule or deplanarising the backbone, also change the shape anisotropy, which may have the opposite effect on interface orientation. Despite these challenges, enhancing the hydrogen-bonding capabilities of a small molecule in the side-side direction has recently been shown to promote a more face-on orientation at a solid substrate, ${ }^{77}$ as predicted.

Increasing the interaction strength of a solid substrate promotes the face-on orientation at the solid interface. Changing the substrate (eg. from silicon to graphite) to one that interacts more strongly with the semiconductor is predicted to shift the orientation towards face-on, corresponding to a shift toward 
the top left of Fig. 6a. There are many examples in the literature of this behaviour. ${ }^{19,44-46,52-55}$ Choosing a substrate that interacts favourably with either OSC backbone or side-chains would also be an effective means of tuning the orientation at this interface.

Processing conditions that favour entropy promote face-on orientation at the solid interface, and side-on at the vapour. As with the shape anisotropy, increasing the effect of entropy by processing at high temperature and pressure (density), will shift the preferred orientation towards the entropically favoured one (face-on at the solid interface, side-on at the vapour). When considering high temperatures, care should be taken to ensure that the orientation remains in the entropically favoured orientation as the system is cooled down, which can be achieved by rapid quenching, as the degree of alignment will be a function of temperature. In cases where the equilibrium orientation is face-on at the solid interface (which appear to be many), processes such as melt annealing can allow the equilibrium orientation to be obtained, even if deposited through a non-equilibrium method in the opposite orientation. 51,78

Conditions for which the bulk fluid is anisotropic, or sideside interactions are stronger than face-face ones, can give the face-on orientation at the vapour interface at equilibrium. From our predictions based on the behaviour of a bulk isotropic fluid, the face-on orientation at the vapour interface is extremely difficult to achieve, although it can be obtained by systems with stronger side-side interactions than face-face interactions (e.g. by chemical functionalisation of the aromatic core of the OSC). However, with face-face interactions typical of OSCs, such systems are unlikely to form isotropic bulk phases under typical conditions. The interface orientation of liquid crystal fluids has previously been shown to be much more variable. ${ }^{36}$ Under appropriate conditions, many OSCs form liquid-crystal phases, meaning the predictions of ref 36 based on the shape and interaction anisotropy can be applied to tune the orientation at the vapour interface.

Non-equilibrium processing can result in different behaviour than that predicted here. The above guidelines apply under equilibrium conditions, but could be circumvented using nonequilibrium methods to potentially reach the more difficult to obtain orientations (face-on at the vapour interface, side-on at the solid interface). Many common device processing techniques are non-equilibrium in nature. In methods such as vapour deposition, the layer-by-layer deposition traps lower layers close to their asdeposited orientation (see refs 79 and 80 for extensive reviews of this method and how processing temperature can influence the orientation). Alternate methods, such as spin or blade coating, rely on the evaporation of solvent to form thin films, which is also inherently a non-equilibrium process and will not necessarily result in the equilibrium structure. Nevertheless, the equilibrium structure is still important for OSCs, since systems tend toward equilibrium over time. For thermal stability of devices it is therefore beneficial for the equilibrium structure to be the one that maximises device performance.

\section{Conclusions}

Through the use of classical simulations of oblate ellipsoidal fluid particles, this work provides a general analysis of how particle shape and interaction anisotropy influence the equilibrium orientation of a wide variety of isotropic liquids at interfaces with both a solid and vapour-like phase. For a range of parameters spanning a subset of organic semiconductor (OSC) space, different substrate types, and different thermodynamic conditions, we find remarkable universal scaling of interface orientation with a simple interfacial free energy parameter based on nearest-neighbour pair interactions and the interfacial entropy of purely repulsive particles of the same shape. Based on these results, we propose several practical methods for achieving the desired orientation at both interfaces, which we hope provide a pathway towards the rational design of high performing OSC-based devices.

At the interface with a solid, the entropically favoured orientation is face-on, consistent with previous theories based on excluded volume entropic effects. Even when attractions are introduced, the orientation is largely influenced by the entropy and can be tuned by modifying the shape anisotropy. The side-on orientation is more difficult to obtain, but can be achieved most simply by using a substrate that interacts weakly with the face of the fluid particle (or strongly with its side).

The entropically favoured orientation at the vapour interface is side-on, in contrast to the alignment at the solid substrate, but again can be rationalised through excluded-volume entropic effects. Importantly, in all cases studied here, the orientation at the vapour interface of a fluid that is isotropic in the bulk is predicted, and generally observed, to be side-on. Conditions for which the face-on orientation is favoured appear to be difficult to achieve for an isotropic bulk liquid phase for interactions typical of OSCs, but can be obtained if the system has a bulk liquid-crystal phase. Tuning of molecular shape and interaction parameters can result in a shift towards a more isotropic interfacial layer.

Many open questions remain in this area, such as how sidechains, biaxiality, degree of polymerisation, multiple fluid components, or non-equilibrium processes such as pre-aggregation of molecules in solution during solvent evaporation, influence the orientation, but we hope that this work provides a useful starting point for understanding some simple methods for how interfacial orientation can be systematically tuned. Additionally, although this work focuses on oblate ellipsoidal particles, representative of molecules such as perylene, similar arguments should be applicable to prolate molecules such as pentacene. Further studies that account for interactions with the surrounding layers may allow this analysis to be extended to the non-isotropic systems that may be more typical of certain OSC molecules.

\section{Conflicts of interest}

There are no conflicts to declare.

\section{Acknowledgements}

This research was undertaken with the assistance of resources from the National Computational Infrastructure (NCI), which is supported by the Australian Government, and from The Univer- 
sity of Adelaide's Phoenix High Performance Computing Service. BJB acknowledges The University of Adelaide for the Joyner and Constance Fraser scholarships and the Playford Memorial Trust for a PhD scholarship.

\section{References}

1 G. A. DiLisi, An Introduction to Liquid Crystals, IOP Publishing, 2019.

2 W. Tang, Y. Huang, L. Han, R. Liu, Y. Su, X. Guo and F. Yan, J. Mater. Chem. C, 2019, 7, 790-808.

3 P. Cheng, G. Li, X. Zhan and Y. Yang, Nat. Photon., 2018, 12, 131-142.

4 M. Kiguchi, M. Nakayama, K. Fujiwara, K. Ueno, T. Shimada and K. Saiki, Jpn. J. Appl. Phys., 2003, 42, L1408.

5 G. Horowitz, J. Mater. Res., 2004, 19, 1946-1962.

6 F. Dinelli, M. Murgia, P. Levy, M. Cavallini, F. Biscarini and D. M. de Leeuw, Phys. Rev. Lett., 2004, 92, 116802.

7 S. M. Menke, N. A. Ran, G. C. Bazan and R. H. Friend, Joule, 2018, 2, 25-35.

8 P. de Gennes and J. Prost, The Physics of Liquid Crystals, Oxford University Press, 1995.

9 H. Sirringhaus, P. J. Brown, R. H. Friend, M. M. Nielsen, K. Bechgaard, B. M. W. Langeveld-Voss, A. J. H. Spiering, R. a. J. Janssen, E. W. Meijer, P. Herwig and D. M. de Leeuw, Nature, 1999, 401, 685-688.

10 X. Zhang, L. J. Richter, D. M. DeLongchamp, R. J. Kline, M. R. Hammond, I. McCulloch, M. Heeney, R. S. Ashraf, J. N. Smith, T. D. Anthopoulos, B. Schroeder, Y. H. Geerts, D. A. Fischer and M. F. Toney, J. Am. Chem. Soc., 2011, 133, 15073-15084.

11 M. M. Nahid, A. Welford, E. Gann, L. Thomsen, K. P. Sharma and C. R. McNeill, Adv. Electron. Mater., 2018, 4, 1700559.

12 C.-F. Liu, Y. Lin, W.-Y. Lai and W. Huang, Mater. Lett., 2017, 189, 286-289.

13 D. Khim, A. Luzio, G. E. Bonacchini, G. Pace, M.-J. Lee, Y.-Y. Noh and M. Caironi, Adv. Mater, 2018, 30, 1705463.

14 M. S. Chen, J. R. Niskala, D. A. Unruh, C. K. Chu, O. P. Lee and J. M. J. Fréchet, Chem. Mater., 2013, 25, 4088-4096.

15 C. Poelking, M. Tietze, C. Elschner, S. Olthof, D. Hertel, B. Baumeier, F. Würthner, K. Meerholz, K. Leo and D. Andrienko, Nat. Mater., 2015, 14, 434-439.

16 J. R. Tumbleston, B. A. Collins, L. Yang, A. C. Stuart, E. Gann, W. Ma, W. You and H. Ade, Nat. Photon., 2014, 8, 385-391.

17 C. Lee, T. Giridhar, J. Choi, S. Kim, Y. Kim, T. Kim, W. Lee, H.H. Cho, C. Wang, H. Ade and B. J. Kim, Chem. Mater, 2017, 29, 9407-9415.

18 L. Ye, X. Jiao, M. Zhou, S. Zhang, H. Yao, W. Zhao, A. Xia, H. Ade and J. Hou, Adv. Mater., 2015, 27, 6046-6054.

19 A. L. Ayzner, D. Nordlund, D.-H. Kim, Z. Bao and M. F. Toney, J. Phys. Chem. Lett., 2015, 6, 6-12.

20 K.-H. Kim, S. Lee, C.-K. Moon, S.-Y. Kim, Y.-S. Park, J.-H. Lee, J. Woo Lee, J. Huh, Y. You and J.-J. Kim, Nat. Commun., 2014, 5, 4769.

21 D. Yokoyama, J. Mater. Chem., 2011, 21, 19187-19202.

22 T. Komino, H. Tanaka and C. Adachi, Chem. Mater., 2014, 26,
3665-3671.

23 T. Lee, B. Caron, M. Stroet, D. M. Huang, P. L. Burn and A. E. Mark, Nano Lett., 2017, 17, 6464-6468.

24 I. Osaka and K. Takimiya, Polymer, 2015, 59, A1-A15.

25 M. Yoneya, S. Matsuoka, J. Tsutsumi and T. Hasegawa, J. Mater. Chem. C, 2017, 5, 9602-9610.

26 K. Bagchi, N. E. Jackson, A. Gujral, C. Huang, M. F. Toney, L. Yu, J. J. de Pablo and M. D. Ediger, J. Phys. Chem. Lett., 2019, 10, 164-170.

27 L. Muccioli, G. D’Avino and C. Zannoni, Adv. Mater., 2011, 23, 4532-4536.

28 Y. Youn, D. Yoo, H. Song, Y. Kang, K. Y. Kim, S. H. Jeon, Y. Cho, K. Chae and S. Han, J. Mater. Chem. C, 2018, 6, 1015-1022.

29 D. M. Walters, L. Antony, J. J. de Pablo and M. D. Ediger, J. Phys. Chem. Lett., 2017, 8, 3380-3386.

30 T. Lee, A. V. Sanzogni, P. L. Burn and A. E. Mark, ACS Appl. Mater. Interfaces, 2020, 12, 40548-40557.

31 B. J. Boehm, H. T. L. Nguyen and D. M. Huang, J. Phys: Condens. Matter, 2019, 31, 423001.

32 M. P. Allen, Mol. Phys., 2019, 117, 2391-2417.

33 H. Kimura and H. Nakano, J. Phys. Soc. Jpn., 1985, 54, 17301736.

34 S. J. Mills, C. M. Care, M. P. Neal and D. J. Cleaver, Phys. Rev. E, 1998, 58, 3284-3294.

35 M. A. Bates, Phys. Rev. E, 2002, 65, 041706.

36 L. F. Rull and J. M. Romero-Enrique, Mol. Phys., 2017, 115, 1214-1224.

37 K. Okano, Jpn. J. Appl. Phys., 1983, 22, L343-L344.

38 H. Reich and M. Schmidt, J. Phys: Condens. Matter, 2007, 19, 326103.

39 L. Harnau and S. Dietrich, Phys. Rev. E, 2002, 65, 021505.

40 A. Poniewierski and R. Hol/yst, Phys. Rev. A, 1988, 38, 37213727.

41 A. Kapanowski and M. Abram, Phys. Rev. E, 2014, 89, 062503.

42 H. Kimura, J. Phys. Soc. Jpn., 1993, 62, 2725-2733.

43 G. D. Wall and D. J. Cleaver, Mol. Phys., 2003, 101, 11051112.

44 T. Breuer, A. Karthäuser and G. Witte, Adv. Mater. Interfaces, 2016, 3, 1500452.

45 D. Salgado-Blanco, C. I. Mendoza, M. A. Chávez-Rojo, J. A. Moreno-Razo and E. Díaz-Herrera, Soft Matter, 2018, 14, 2846-2859.

46 T. Wang, T. R. Kafle, B. Kattel, Q. Liu, J. Wu and W.-L. Chan, Sci. Rep., 2016, 6, 28895.

47 A. Pizzirusso, R. Berardi, L. Muccioli, M. Ricci and C. Zannoni, Chem. Sci., 2012, 3, 573-579.

48 O. M. Roscioni, L. Muccioli, R. G. Della Valle, A. Pizzirusso, M. Ricci and C. Zannoni, Langmuir, 2013, 29, 8950-8958.

49 O. M. Roscioni, L. Muccioli and C. Zannoni, ACS Appl. Mater. Interfaces, 2017, 9, 11993-12002.

50 O. Dolynchuk, P. Schmode, M. Fischer, M. Thelakkat and T. Thurn-Albrecht, Macromolecules, 2021, 54, 5429-5439.

51 N. Boulanger, V. Yu, M. Hilke, M. F. Toney and D. R. Barbero, 
Phys. Chem. Chem. Phys., 2017, 19, 8496-8503.

52 I. Salzmann, A. Moser, M. Oehzelt, T. Breuer, X. Feng, Z.Y. Juang, D. Nabok, R. G. Della Valle, S. Duhm, G. Heimel, A. Brillante, E. Venuti, I. Bilotti, C. Christodoulou, J. Frisch, P. Puschnig, C. Draxl, G. Witte, K. Müllen and N. Koch, ACS Nano, 2012, 6, 10874-10883.

53 R. Guo, B. Li, T. Lu, T. Lin, J. Andre, C. Zhang, L. Zhi and Z. Chen, Macromolecules, 2021, 54, 4050-4060.

54 M. Shahiduzzaman, T. Hirayama, T. Chikamatsu, T. Koganezawa, M. Nakano, T. Miyadera, M. Karakawa, K. Takahashi and T. Taima, Mol. Syst. Des. Eng., 2020, 5, 559-564.

55 M. Beigmohamadi, P. Niyamakom, A. Farahzadi, C. Effertz, S. Kremers, D. Brueggemann and M. Wuttig, J. Appl. Phys., 2008, 104, 013505.

56 P. I. C. Teixeira, C. Anquetil-Deck and D. J. Cleaver, Liq. Cryst., 2021, 48, 75-87.

57 C. Anquetil-Deck, D. J. Cleaver and P. I. C. Teixeira, J. Phys. Chem. B, 2020, 124, 7709-7716.

58 S. J. Plimpton, J. Comput. Phys., 1995, 117, 1-19.

59 W. M. Brown, M. K. Petersen, S. J. Plimpton and G. S. Grest, J. Chem. Phys., 2009, 130, 044901.

60 A. Stukowski, Modelling Simul. Mater. Sci. Eng., 2009, 18, 015012.

61 J. G. Gay and B. J. Berne, J. Chem. Phys., 1981, 74, 33163319.

62 R. Berardi, C. Fava and C. Zannoni, Chem. Phys. Lett., 1998, 297, 8-14.

63 W. L. Jorgensen, D. S. Maxwell and J. Tirado-Rives, J. Am. Chem. Soc., 1996, 118, 11225-11236.

64 C. Lorenz, E. Webb, M. Stevens, M. Chandross and G. Grest,
Tribol. Lett., 2005, 19, 93-98.

65 P. Becker, P. Scyfried and H. Siegert, Z. Phys. B Condens. Matter, 1982, 48, 17-21.

66 S. Nosé, J. Chem. Phys., 1984, 81, 511-519.

67 W. G. Hoover, Phys. Rev. A, 1985, 31, 1695-1697.

68 M. R. Shirts and J. D. Chodera, J. Chem. Phys., 2008, 129, 124105.

69 J. D. Chodera, W. C. Swope, J. W. Pitera, C. Seok and K. A. Dill, J. Chem. Theory Comput., 2007, 3, 26-41.

70 J. D. Chodera, J. Chem. Theory Comput., 2016, 12, 17991805.

71 L. F. Rull, Physica A, 1995, 220, 113-138.

72 J. D. Weeks, D. Chandler and H. C. Andersen, J. Chem. Phys., 1971, 54, 5237-5247.

73 D. L. Cheung, J. Chem. Phys., 2008, 128, 194902.

74 J. C. Maunoury, J. R. Howse and M. L. Turner, Adv. Mater., 2007, 19, 805-809.

75 B.-G. Kim, E. J. Jeong, J. W. Chung, S. Seo, B. Koo and J. Kim, Nat. Mater., 2013, 12, 659-664.

76 L. Yang, J. R. Tumbleston, H. Zhou, H. Ade and W. You, Energy Environ. Sci., 2012, 6, 316-326.

77 A. D. Nicola, A. Correa, A. Giunchi, L. Muccioli, G. D'Avino, J. Kido and G. Milano, Adv. Theory Simul., 2021, 4, 2000302.

78 V. Skrypnychuk, N. Boulanger, V. Yu, M. Hilke, M. F. Toney and D. R. Barbero, J. Mater. Chem. C, 2016, 4, 4143-4149.

79 K. Bagchi and M. D. Ediger, J. Phys. Chem. Lett., 2020, 11, 6935-6945.

80 A. Gujral, L. Yu and M. D. Ediger, Curr. Opin. Solid State Mater. Sci., 2018, 22, 49-57. 